\title{
ON HYPERSINGULAR INTEGRALS AND CERTAIN SPACES OF LOCALLY DIFFERENTIABLE FUNCTIONS
}

\author{
BY \\ RICHARD L. WHEEDEN
}

1. Introduction. In this paper, we shall study relations between pointwise convergence of hypersingular integrals and local differential properties of functions. Our results will partly generalize a theorem of Calderón and Zygmund and an unpublished theorem of E. M. Stein.

We will use standard notation for points and functions in $n$-dimensional Euclidean space $E^{n}, n \geqq 2$. If $f(x) \in L^{p}\left(E^{n}\right), 1 \leqq p<\infty$, set

$$
f_{\varepsilon}(x)=\int_{|z|>8}[f(x-z)-f(x)] \frac{\Omega\left(z^{\prime}\right)}{|z|^{n+\alpha}} d z
$$

for $\varepsilon>0$ and $0<\alpha<2$, where $\Omega$ is a bounded real-valued function homogeneous of degree zero which satisfies

$$
\int_{\Sigma} z_{j}^{\prime} \Omega\left(z^{\prime}\right) d z^{\prime}=0 \quad(j=1, \ldots, n)
$$

for $1 \leqq \alpha<2$. Here $\Sigma$ denotes the unit sphere of points $z^{\prime}=z /|z|, z \neq 0$.

If $\lim _{\varepsilon \rightarrow 0} f_{\varepsilon}(x)$ exists in some sense, we call it a hypersingular integral of $f$. If, for example, $f$ satisfies the global differentiability condition $f \in L_{\alpha}^{p}=J^{\alpha} L^{p}$ (see [2]), the convergence of $f_{\varepsilon}$ in various senses was studied in [8], [14] and [15]. Thus far, however, the convergence of $f_{\varepsilon}$ for $f$ satisfying a local differentiability condition has been studied for $n \geqq 2$ only in case $\alpha=1$. (See [5].)

Following [4], we say an $f \in L^{p}, 1 \leqq p<\infty$, belongs to $t_{\alpha}^{p}\left(x_{0}\right)$ if there is a polynomial $P_{x_{0}}(z)$ of degree less than or equal to $\alpha$ such that

$$
\left(\varepsilon^{-n} \int_{|z|<\varepsilon}\left|f\left(x_{0}+z\right)-P_{x_{0}}(z)\right|^{p} d z\right)^{1 / p}=o\left(\varepsilon^{\alpha}\right)
$$

as $\varepsilon \rightarrow 0$. We say $f \in T_{\alpha}^{p}\left(x_{0}\right)$ if there is a polynomial of degree strictly less than $\alpha$ such that

for $\varepsilon>0$.

$$
\left(e^{-n} \int_{|z|<8}\left|f\left(x_{0}+z\right)-P_{x_{0}}(z)\right|^{p} d z\right)^{1 / p}=O\left(e^{\alpha}\right)
$$

Given $0<\alpha<2$ let $\beta=[\alpha]+1-\alpha$, so that $\alpha+\beta=1$ if $0<\alpha<1$ and $\alpha+\beta=2$ if $1 \leqq \alpha<2$. Roughly speaking, our main result is that for $f \in T_{\alpha}^{p}(x)$ the convergence

Received by the editors June 7, 1968 and, in revised form, June 19, 1969.

Copyright (C) 1969 , American Mathematical Society 
of $f_{\varepsilon}(x)$ is equivalent almost everywhere to the condition $J^{\beta} f \in T_{\alpha+\beta}^{p}(x)$. Calderón and Zygmund show in [4] (Theorems 4 and 5) that if $f \in T_{\alpha}^{p}(x)$ for $x \in E$ then $J^{\gamma} f \in T_{\alpha+\gamma}^{p}(x)$ for almost every $x \in E$, except in the special case that $\alpha+\gamma$ is an integer but $\alpha$ and $\gamma$ are not. This is precisely our case, however, and an example of the complications which may arise can be found in [16, pp. 136-138].

We shall prove the following results:

THEOREM 1. Given $0<\alpha<2$, let $\Omega$ be a bounded function homogeneous of degree zero which satisfies (1.1) when $1 \leqq \alpha<2$. Let $f \in L^{p}, 1 \leqq p<\infty, E \subset E^{n}$ and $\beta=[\alpha]$ $+1-\alpha$. If $f \in T_{\alpha}^{p}(x)$ for $x \in E$ and $J^{\beta} f \in T_{\alpha+\beta}^{p}(x)$ for $x \in E$ then

$$
\lim _{\varepsilon \rightarrow 0} \int_{|z|>\varepsilon}[f(x-z)-f(x)] \frac{\Omega\left(z^{\prime}\right)}{|z|^{n+\alpha}} d z
$$

exists and is finite for almost all $x \in E$.

Conversely,

THEOREM 2. Let $f \in L^{p}, 1 \leqq p<\infty, E \subset E^{n}, 0<\alpha<2$. Suppose $f \in T_{\alpha}^{p}(x)$ for $x \in E$ and each

$$
\int_{|z|>\varepsilon}[f(x-z)-f(x)] \frac{\Omega_{j}(z)}{|z|^{n+\alpha}} d z
$$

converges for $x \in E$, where $\left\{\Omega_{j}\right\}$ is a basis for the spherical harmonics of a fixed degree $m \geqq 0, m \neq 1$ when $1 \leqq \alpha<2$ and $m=0$ when $p=1$. Then with $\beta=[\alpha]+1-\alpha$, $J^{\beta} f \in T_{\alpha+\beta}^{p}(x)$ for almost all $x \in E$.

When $\alpha=1$, the hypothesis $f \in T_{1}^{p}(x)$ for $x \in E$ implies that $f \in t_{1}^{p}(x)$ and $J^{1} f \in t_{2}^{p}(x)$ for almost all $x \in E\left(^{1}\right)$ and Theorem 1 is a known result of Calderón and Zygmund [5]. Also, Theorem 2 for $\alpha=1$ is vacuous and a replacement result is the following.

THEOREM 3. Let $f \in L^{p}, 1 \leqq p<\infty, E \subset E^{n}$. If

$$
\left(\varepsilon^{-n} \int_{|z|<\varepsilon}|f(x+z)+f(x-z)-2 f(x)|^{p} d z\right)^{1 / p}=O(\varepsilon)
$$

for $x \in E$ and each

$$
\int_{|z|>\varepsilon}[f(x-z)-f(x)] \frac{\Omega_{y}(z)}{|z|^{n+1}} d z
$$

converges for $x \in E$, where the $\Omega$, are as in Theorem 2 , then $f \in t_{1}^{p}(x)$ for almost all $x \in E$.

Theorem 3 for $\Omega \equiv 1$ was proved independently by E. M. Stein. It turns out that for all $0<\alpha<2$ one can replace the condition $f \in T_{\alpha}^{p}(x)$ of Theorem 2 by an apparently weaker condition. See the remark at the end of $\$ 4$.

(1) See Theorem 5 of [4]. Although Theorems 4 and 5 are stated for $p>1$, it is not hard to see they remain true for $p=1$ when, with the notation of [4], $q=1$ and $u>0$. 
Although we stated our theorems for $n \geqq 2$ they have analogues for $n=1$ which are related to the results of [13]. In their present form, our results do not include those of Sagher [7] for hypersingular integrals with complex homogeneity.

We shall prove Theorem 1 in $\S 2$, Theorem 2 in $\S 3$ and Theorem 3 in $\S 4$. $\S 4$ also contains an apparent improvement of Theorem 2.

2. Proof of Theorem 1. We will use the method in [11] to prove Theorem 1. We need a long list of lemmas, and in order to shorten their presentation we will assume $1<\alpha<2$ whenever convenient. We also note that it suffices to prove Theorem 1 for $p=1$ since we may assume $E$ is bounded and $f$ has compact support and since the condition $f \in T_{\alpha}^{p}(x)$ for $p>1$ implies $f \in T_{\alpha}^{1}(x)$.

We recall that $f \in L_{\alpha}^{p}, 1 \leqq p<\infty, \alpha>0$, if $f=J^{\alpha} \phi=G_{\alpha} * \phi$ for $\phi \in L^{p}$ where $G_{\alpha}$ is a positive integrable function with the following properties (see e.g. [4]):

(a) $\hat{G}_{\alpha}(x)=\left(1+|x|^{2}\right)^{-\alpha / 2}$,

(b) $G_{\alpha}$ is infinitely differentiable except at $x=0$ and for $x \neq 0,0<\alpha<n$ and $|\nu| \geqq 0$

$$
\left|\left(\partial^{\nu} / \partial x^{\nu}\right) G_{\alpha}(x)\right| \leqq c_{\alpha, \nu} e^{-|x|}\left[1+|x|^{-n-|\nu|+\alpha}\right] .
$$

LEMMA 1. Let $f \in L_{\alpha}^{p}$ for some $0<\alpha<2$ and let $\Omega$ satisfy the hypothesis of Theorem 1. Then for $1 \leqq p<\infty$

$$
f(x)=\lim _{8 \rightarrow 0} \int_{|z|>8}[f(x-z)-f(x)] \frac{\Omega\left(z^{\prime}\right)}{|z|^{n+\alpha}} d z
$$

exists and is finite for almost all $x$, and for $1<p<\infty$, the transformation $f \rightarrow f$ sends $L_{\alpha}^{p}$ boundedly into $L^{p}$.

For a proof, see [15].

LEMMA 2. Given $\lambda>0$,

(a) $|x|^{\lambda}=\left(1+|x|^{2}\right)^{\lambda / 2} d \hat{\mu}(x)$,

(b) $\left(1+|x|^{2}\right)^{\lambda / 2}=|x|^{\lambda} d \hat{\sigma}(x)+d \hat{\tau}(x)$,

where dî is the sum of 1 , a finite linear combination of terms $\hat{G}_{2 k}, k=1,2, \ldots$, and the Fourier transform of a function with derivatives up to a preassigned order belonging to all $L^{p}, 1 \leqq p \leqq \infty, d \hat{\sigma}$ is the sum of 1 and a finite linear combination of terms $\hat{G}_{2 k}, k=1,2, \ldots$, and $d \hat{\tau}$ is the Fourier transform of a function with derivatives up to a preassigned order belonging to all $L^{p}, 1 \leqq p \leqq \infty$.

Parts (a) and (b) of Lemma 2 are stated in [8]. The proof of the rest of the lemma is not difficult and we omit it.

LEMMA 3. Let $f \in L^{1}, 1<\alpha<2, \alpha+\beta=2, F=J^{\beta} f$. Then for almost all $x$,

$$
f(x)=c F_{\tau}(x)+c_{\beta} \int\left[F_{\sigma}(x+z)-F(x)\right] \frac{d z}{|z|^{n+\beta}}
$$

where $F_{\sigma}=F * d \sigma, F_{\tau}=F * d \tau, d \sigma$ and $d \tau$ being defined by Lemma 2 with $\lambda=\beta$. 
The integral in (2.1) exists almost everywhere in the principal value sense by Lemma 1 since $F_{\sigma} \in L_{\beta}^{1}$. Moreover, by Lemma (1.6) of [14],

$$
c_{\beta} \int_{|z|>\varepsilon}\left[F_{\sigma}(x+z)-F_{\sigma}(x)\right] \frac{d z}{|z|^{n+\beta}}-\int_{E^{n}} F_{\sigma}(x+z)\left[|z|^{\beta} e^{-\varepsilon|z|}\right]^{\wedge} d z
$$

tends to zero with $\varepsilon$ for almost all $x$. Hence the right side of (2.1) is the limit almost everywhere of

$$
\begin{aligned}
\int_{E^{n}} F_{z}(x+z)\left[e^{-\varepsilon|z|}\right]^{\wedge} d z+\int_{E^{n}} F_{\sigma}( & x+z)\left[|z|^{\beta} e^{-\varepsilon|z|}\right]^{\wedge} d z \\
& =c \int \hat{F}(z)\left[|z|^{\beta} d \hat{\sigma}(z)+d \hat{\tau}(z)\right] e^{\{(x \cdot z)} e^{-\varepsilon|z|} d z \\
& =c \int \hat{f}(z) e^{\{(x \cdot z)} e^{-\varepsilon|z|} d z
\end{aligned}
$$

by Lemma 2(b) and the fact that $\hat{F}(z)=\left(1+|z|^{2}\right)^{-\beta / 2} \hat{f}(z)$. The last integral is essentially the Poisson integral of $f$ and converges to a constant times $f$ almost everywhere.

LEMMA 4. If $f \in L^{1}$ and $\alpha>0$ is not an integer then $J^{\alpha} f \in t_{\alpha}^{1}(x)$ for almost all $x$.

The proof of Lemma 4 is almost identical to that of Theorem 4 of [4]. Although the case $p=1$ is not considered there, the proof easily yields Lemma 4. (See also the proof of Lemma 4 of $\$ 3$ below.)

LEMMA 5. Let $1<\alpha<2$ and $v(x)$ and its first order derivatives be continuous and have compact support. For any $j=1, \ldots, n$,

$$
u(x)=\int_{E^{n}} v(x-z) \frac{z_{j}^{\prime}}{|z|^{n-(\alpha-1)}} d z
$$

belongs to $T_{\alpha}^{1}(x)$ uniformly in $x$.

Proof. If $u_{i}=\left(\partial / \partial x_{i}\right) u$ then

$$
u_{i}(x)=\int_{E^{n}} v_{i}(x-z) \frac{z_{j}^{\prime}}{|z|^{n-(\alpha-1)}} d z
$$

is continuous and

$$
\begin{aligned}
\left|u_{i}(x+y)-u_{i}(x)\right| \leqq & c \int\left|\frac{1}{|z+y|^{n-(\alpha-1)}}-\frac{1}{|z|^{n-(\alpha-1)}}\right| d z \\
& +c \int \frac{1}{|z|^{n-(\alpha-1)}}\left|(z+y)_{j}^{\prime}-z_{j}^{\prime}\right| d z .
\end{aligned}
$$

Each integral is easily seen to be $O\left(|y|^{\alpha-1}\right)$ and the lemma follows from Taylor's formula. 
The remaining lemmas are taken from [4].

LEMMA 6. Let $P$ be a closed subset of $E^{n}$ and $U$ be the neighborhood of $P$ of all points whose distance from $P$ is less than 1. Then there is a covering of $U-P$ by nonoverlapping closed cubes $K_{m}$ with $c^{-1} \leqq d_{m} / e_{m} \leqq c, 0<c<\infty$, where $e_{m}$ is the edge length of $K_{m}$ and $d_{m}$ is the distance from $K_{m}$ to $P$.

See Lemma (3.1) of [4].

LEMMA 7. Let $P$ be a compact set and $\delta(x)$ be the distance from $x$ to $P$, with $\delta(x)=0$ for large $x$. Given $\lambda>0$

$$
\int_{E^{n}} \frac{\delta^{\lambda}(x+z)}{|z|^{n+\lambda}} d z
$$

is finite for almost all $x \in P$.

LEMMA 8. Let $F \in t_{2}^{1}(x)$ for $x \in E, E$ a bounded measurable set. Given $\varepsilon>0$ there is a closed set $P \subset E,|E-P|<\varepsilon$, and a decomposition $F=G+H$ where $G$ has two continuous derivatives and compact support, $H(x)=0$ for $x \in P$ and

$$
\int_{|z|<\varepsilon}|H(x+z)| d z \leqq M \varepsilon^{n+2}
$$

uniformly for $x \in P$. Moreover, given $0<\lambda \leqq 2$,

$$
\int \frac{|H(z)|}{|x-z|^{n+\lambda} \delta(z)^{2-\lambda}} d z
$$

is finite for almost all $x \in P, \delta(z)$ being the distance from $z$ to $P$.

Integration in (2.3) is of course extended over the complement of $P$. Lemma 8 for $\lambda=2$ is proved in [4, p. 189-190], and the proof for $0<\lambda \leqq 2$ is similar.

LEMMA 9. Let $h \in T_{\alpha}^{1}(x), 1<\alpha<2$, uniformly for $x$ in a closed set $P$, i.e.,

$$
\varepsilon^{-n} \int_{|z|<8}\left|h(x+z)-h(x)-\sum z_{j} h_{j}(x)\right| d z \leqq M \varepsilon^{\alpha}
$$

for $x \in P$. Then for $x$ and $x+z$ in $P$,

and

$$
\left|h(x+z)-h(x)-\sum z_{j} h_{j}(x)\right| \leqq M^{\prime}|z|^{\alpha}
$$

$$
\left|h_{j}(x+z)-h_{f}(x)\right| \leqq M^{\prime}|z|^{\alpha-1} \quad(j=1, \ldots, n) .
$$

We can now prove Theorem 1 for $1<\alpha<2$. Let $f$ and $F=J^{\beta} f, \beta=2-\alpha$, satisfy the hypothesis of Theorem 1 for $p=1$. Let $F_{\sigma}$ and $F_{\tau}$ be defined as in Lemma 3. Since $F \in L^{1}$ and $F_{\tau}$ is a convolution of $F$ with a function with bounded derivatives 
up to a preassigned order (Lemma 2), we may assume $F_{\tau}$ has bounded continuous second order derivatives everywhere. In particular,

$$
\left|F_{\tau}(x-z)-F_{\tau}(x)+\sum z_{j}\left(\frac{\partial F_{\tau}}{\partial x_{j}}\right)(x)\right| \leqq M|z|^{2},
$$

for all $x$ and $z, M<\infty$. Since $1<\alpha<2$,

$$
\int\left[F_{\tau}(x-z)-F_{\tau}(x)+\sum z_{j}\left(\frac{\partial F_{\tau}}{\partial x_{j}}\right)(x)\right] \frac{\Omega\left(z^{\prime}\right)}{|z|^{n+\alpha}} d z
$$

converges absolutely everywhere. Since $\Omega$ is orthogonal to polynomials of degree 1 ,

$$
\int_{|z|>\varepsilon}\left[F_{z}(x-z)-F_{z}(x)\right] \frac{\Omega\left(z^{\prime}\right)}{|z|^{n+\alpha}} d z
$$

converges everywhere as $\varepsilon \rightarrow 0$.

Hence, applying (2.1), it remains to prove the conclusion of Theorem 1 with $f$ replaced by

$$
\text { p.v. } \int\left[F_{\sigma}(x+z)-F_{\sigma}(x)\right] \frac{d z}{|z|^{n+\beta}} \text {. }
$$

By (2.4) $F_{\tau} \in T_{\alpha}^{1}(x)$ everywhere and by (2.1) again, the same is true for $x \in E$ of (2.5). By Lemma 2, $F_{\sigma}$ is the sum of $F$ and a finite linear combination of terms $J^{2 k} F, k \geqq 1$. It follows that $F_{\sigma} \in t_{2}^{1}(x)$ for almost all $x \in E$. Here we use first the fact, noted in $\S 1$, that $T_{2}^{1}(x)$ and $t_{2}^{1}(x)$ are equivalent almost everywhere and next the fact that $J^{2 k} F=J^{2 k+\beta} f \in t_{2 k+\beta}^{1}(x)$ for almost all $x$ (Lemma 4). Since $k \geqq 1$,

$$
t_{2 k+\beta}^{1}(x) \subset t_{2}^{1}(x) .
$$

Collecting these facts, we see it is enough to prove Theorem 1 for $f \in T_{\alpha}^{1}(x)$, $x \in E$, of the form

$$
f(x)=\text { p.v. } \int_{E^{n}}[F(x+z)-F(x)] \frac{d z}{|z|^{n+\beta}}
$$

where $F \in L_{\beta}^{1}$ and $F \in t_{2}^{1}(x)$ for $x \in E$. For such $F$, form the decomposition $F=G+H$ of $F$ relative to a closed set $P \subset E$ (Lemma 8). We may assume $f \in T_{\alpha}^{1}(x)$ uniformly for $x \in P$. Consider

$$
\begin{aligned}
\int_{|z|>\varepsilon}[G(x+z)-G(x)] \frac{d z}{|z|^{n+\beta}} & =\frac{1}{\alpha-2} \int_{\varepsilon}^{\infty} \frac{d}{d t}\left(t^{\alpha-2}\right) d t \int_{\Sigma}\left[G\left(x+t z^{\prime}\right)-G(x)\right] d z^{\prime} \\
= & \frac{1}{\alpha-2}\left(\left.t^{\alpha-2} \int_{\Sigma}\left[G\left(x+t z^{\prime}\right)-G(x)\right] d z^{\prime}\right|_{\delta} ^{\infty}\right. \\
& \left.\quad-\sum_{j=1}^{n} \int_{\varepsilon}^{\infty} \frac{d t}{t^{2-\alpha}} \int_{\Sigma} z_{j}^{\prime} G_{j}\left(x+t z^{\prime}\right) d z^{\prime}\right),
\end{aligned}
$$


where $G_{j}=\left(\partial / \partial x_{j}\right) G$. At $t=\infty$ the integrated term is zero since $\alpha-2<0$. At $t=\varepsilon$ it is $O\left(\varepsilon^{\alpha-1}\right)=o(1)$. Hence

$$
\begin{aligned}
g(x) & =\lim _{\varepsilon \rightarrow 0} \int_{|z|>\varepsilon}[G(x+z)-G(x)] \frac{d z}{|z|^{n+\beta}} \\
& =\frac{1}{\alpha-2} \sum_{j=1}^{n} \int_{E^{n}} G_{j}(x-z) \frac{z_{j}^{\prime}}{|z|^{n-(\alpha-1)}} .
\end{aligned}
$$

By Lemma 5, $g \in T_{\alpha}^{1}(x)$ uniformly in $x$ for all $x$. Hence $h=f-g \in T_{\alpha}^{1}(x)$ uniformly for $x \in P$. Moreover,

$$
h(x)=f(x)-g(x)=\lim _{8 \rightarrow 0} \int_{|z|>8}[H(x+z)-H(x)] \frac{d z}{|z|^{n+\beta}}
$$

almost everywhere. Since $H=0$ in $P$ and (2.3) with $\lambda=2$ is finite for almost all $x \in P$,

$$
h(x)=\int_{E^{n}} \frac{H(x+z)}{|z|^{n+\beta}} d z
$$

for almost all $x \in P$, the integral converging absolutely.

To prove Theorem 1, it suffices to show that both

and

$$
\tilde{g}_{\varepsilon}(x)=\int_{|z|>\varepsilon}[g(x-z)-g(x)] \frac{\Omega\left(z^{\prime}\right)}{|z|^{n+\alpha}} d z
$$

$$
\tilde{h}_{\varepsilon}(x)=\int_{|z|>\varepsilon}[h(x-z)-h(x)] \frac{\Omega\left(z^{\prime}\right)}{|z|^{n+\alpha}} d z
$$

converge for almost all $x \in P$. Consider first $\tilde{g}_{\varepsilon}$. Since $G \in L_{2}^{2}=J^{\alpha} L_{\beta}^{2}$, it follows from (2.6) and Lemma 1 with $\Omega \equiv 1$ that $g \in L_{\alpha}^{2}$. By Lemma 1 again, $\tilde{g}_{\varepsilon}(x)$ converges for almost all $x$.

Turning to $\tilde{h}_{\varepsilon}$, we have $h \in T_{\alpha}^{1}(x)$, uniformly for $x \in P$, i.e., for $x \in P$

$$
\int_{|z|<\varepsilon}\left|h(x+z)-h(x)-\sum z_{j} h_{j}(x)\right| d z \leqq M \varepsilon^{n+\alpha}
$$

for certain $h_{j}(x)$. We claim that

$$
h_{j}(x)=\int_{E^{n}} H(x-z) \frac{\partial}{\partial z_{j}}\left(\frac{1}{|z|^{n+\beta}}\right) d z
$$

for almost all $x \in P$. Observe that the integral in (2.8) converges absolutely for almost all $x \in P$ since $\alpha>1$ (see (2.3) with $\lambda=2$ ). By Lemma $9, h_{j}(x)$ is the derivative of $h$ with respect to $x_{j}$ restricted to $P$, i.e., if $\varepsilon_{j}=(0, \ldots, 0, \varepsilon, 0, \ldots, 0)$ with $\varepsilon$ as the $j$ th entry, then

$$
(h(x+\varepsilon)-h(x)) / \varepsilon \rightarrow h_{j}(x)
$$


as $\varepsilon \rightarrow 0$ provided $x, x+\varepsilon_{j} \in P$. On the other hand since we may assume (2.7) holds for all $x \in P$, we have for $x$ and $x+\varepsilon_{j}$ in $P$

$$
\begin{array}{rl}
\frac{h\left(x+\varepsilon_{j}\right)-h(x)}{\varepsilon}-\int_{E^{n}} & H(x-z) \frac{\partial}{\partial z_{j}}\left(\frac{1}{|z|^{n+\beta}}\right) d z \\
& =\frac{1}{\varepsilon} \int_{E^{n}} H(x-z)\left[\frac{1}{\left|z+\varepsilon_{j}\right|^{n+\beta}}-\frac{1}{|z|^{n+\beta}}-\varepsilon \frac{\partial}{\partial z_{j}} \frac{1}{|z|^{n+\beta}}\right] d z \\
& =\frac{1}{\varepsilon} \int_{|z|<2 \varepsilon}+\frac{1}{\varepsilon} \int_{|z|>2 \varepsilon}=A_{\varepsilon}+B_{\varepsilon} .
\end{array}
$$

By the mean-value theorem,

$$
\left|B_{\varepsilon}\right| \leqq c \varepsilon \int_{|z|>2 \varepsilon}|H(x+z)| \frac{d z}{|z|^{n+\beta+2}}
$$

If $R(t)=\int_{|z|<t}|H(z+z)| d z$, then $R(t) \leqq M t^{n+2}$ by Lemma 8 and

$$
\left|B_{\varepsilon}\right| \leqq c \varepsilon \int_{2 \varepsilon}^{\infty} \frac{d R(t)}{t^{n+\beta+2}}
$$

Integrating by parts, $B_{\varepsilon}=O\left(\varepsilon^{\alpha-1}\right)=o(1)$.

Even simpler estimates show that the terms

$$
\frac{1}{\varepsilon} \int_{|z|<2 \varepsilon} H(x+z) \frac{d z}{|z|^{n+\beta}} \text { and } \int_{|z|<2 \varepsilon} H(x+z) \frac{\partial}{\partial z j}\left(\frac{1}{|z|^{n+\beta}}\right) d z
$$

of $A_{\varepsilon}$ tend to zero with $\varepsilon$. The remaining term of $A_{\varepsilon}$ is majorized by

$$
\frac{1}{\varepsilon} \int_{|z|<3 \varepsilon}\left|H\left(x+\varepsilon_{j}+z\right)\right| \frac{d z}{|z|^{n+\beta}}=\frac{1}{\varepsilon} \int_{0}^{3 \varepsilon} \frac{d R_{\varepsilon}(t)}{t^{n+\beta}}
$$

where $R_{\varepsilon}(t)=\int_{|z|<t}\left|H\left(x+\varepsilon_{j}+z\right)\right| d z \leqq M t^{n+2}$ uniformly in $\varepsilon$. That (2.8) holds for almost all $x \in P$ now follows by integrating by parts.

We claim next that

$$
\int_{E^{n}}\left|h(x+z)-h(x)-\sum z_{j} h_{j}(x)\right| \frac{d z}{|z|^{n+\alpha}}<\infty
$$

for almost all $x \in P$. Since $\Omega$ is bounded and orthogonal to polynomials of degree 1 , $\lim _{\varepsilon \rightarrow 0} h_{\varepsilon}(x)$ exists wherever (2.9) holds. If we assume that (2.7) and (2.8) hold for all $x \in P$, it is enough to show that (2.9) holds for each point of density $x$ of $P$ at which (2.2) is finite for $\lambda=\alpha$ and $\lambda=1$ and at which (2.3) is finite for $\lambda=\alpha$ and $\lambda=2$. Let $x=0$ be such a point. Then (2.9) for $x=0$ will follow if

$$
\int_{|z|<\eta}\left|h(z)-h(0)-\sum z_{j} h_{j}(0)\right| \frac{d z}{|z|^{n+\alpha}}
$$

is finite for some $\eta>0$. In what follows we will denote by $c$ a constant, possibly different in different occurrences, depending only on $\alpha$ and $n$. 
Consider first that part of (2.10) with integration extended only over $P$. Applying (2.7), (2.8) and interchanging the order of integration,

$$
\begin{aligned}
\int_{P} \mid h(z) & -h(0)-\sum z_{j} h_{j}(0) \mid \frac{d z}{|z|^{n+\alpha}} \\
& \leqq \int|H(y)| d y \int_{P}\left|\frac{1}{|y-z|^{n+\beta}}-\frac{1}{|y|^{n+\beta}}+\sum z_{j} \frac{\partial}{\partial y_{j}}\left(\frac{1}{|y|^{n+\beta}}\right)\right| \frac{d z}{|z|^{n+\alpha}} .
\end{aligned}
$$

By the mean-value theorem, the inner integral extended over $|z|<|y| / 2$ is majorized by a constant times

$$
\int_{|z|<|y| / 2} \frac{|z|^{2}}{|y|^{n+\beta+2}} \frac{d z}{|z|^{n+\alpha}}=O\left(|y|^{-n-2}\right) .
$$

Since (2.3) with $\lambda=2$ and $x=0$ is finite, we may consider the inner integral above extended over $|z|>|y| / 2$. Since $\alpha>1$,

$$
\begin{aligned}
\int|H(y)| d y \int_{|z|>|y| / 2} & \left|z_{j} \frac{\partial}{\partial y_{j}}\left(\frac{1}{|y|^{n+\beta}}\right)\right| \frac{d z}{|z|^{n+\alpha}} \\
& \leqq c \int \frac{|H(y)|}{|y|^{n+\beta+1}} d y \int_{|z|>|y| / 2} \frac{d z}{|z|^{n+\alpha-1}}=c \int \frac{|H(y)|}{|y|^{n+2}} d y .
\end{aligned}
$$

The part

$$
\int|H(y)| d y \int_{|z|>|y| / 2} \frac{1}{|y|^{n+\beta}} \frac{d z}{|z|^{n+\alpha}}
$$

can be treated similarly. Since $H=0$ in $P$ and $|z-y| \geqq \delta(y)$ for $z \in P$ and $y \in P^{\prime}$, the remaining part

$$
\begin{aligned}
\int|H(y)| d y \int_{P ;|z|>|y| / 2} \frac{1}{|y-z|^{n+\beta}} \frac{d z}{|z|^{n+\alpha}} & \leqq c \int_{P^{\prime}} \frac{|H(y)|}{|y|^{n+\alpha}} d y \int_{|y-z|>\delta(y)} \frac{d z}{|y-z|^{n+\beta}} \\
& \leqq c \int \frac{H(y)}{|y|^{n+\alpha} \delta(y)^{2-\alpha}} d y<\infty .
\end{aligned}
$$

Now consider the part of (2.10) with integration extended over $P^{\prime}$. For any $z$, write $\omega(z)=h(z)-h(0)-\sum z_{j} h_{j}(0)$. With the notation of Lemma 6 , let $p_{m} \in P$ be a point whose distance from each point of $K_{m}$ is less than a constant (independent of $m$ ) times $d_{m}$. It is enough to show both

$$
\sum_{m} \int_{K_{m}}\left|\omega(z)-\omega\left(p_{m}\right)\right| \frac{d z}{|z|^{n+\alpha}}
$$

and

$$
\sum_{m} \int_{K_{m}}\left|\omega\left(p_{m}\right)\right| \frac{d z}{|z|^{n+\alpha}}
$$

are finite, summations being extended over all $m$ for which $K_{m}$ intersects $\{z:|z|<\eta\}$.

Let $\delta_{m}$ be the distance from $K_{m}$ to 0 . Since 0 is a point of density of $P$ we can choose $\eta$ so small that $\left|p_{m}\right| \leqq c \delta_{m}$ and $\delta_{m} \leqq|z| \leqq c \delta_{m}$ for $z \in K_{m}$, with $c$ independent 
of $m$. Fix $m$ and write $K=K_{m}, p=p_{m}$, etc. A term of (2.11) is then majorized by a constant independent of $m$ times

$$
\delta^{-n-\alpha} \int_{K}\left|h(z)-h(p)-\sum_{j}\left(z_{j}-p_{j}\right) h_{j}(p)\right| d z+d \delta^{-n-\alpha} \sum_{j} \int_{K}\left|h_{j}(p)-h_{j}(0)\right| d z .
$$

If we replace integration over $K$ by integration over $|z-p|<c d$, we only increase this. Moreover, $h \in T_{\alpha}^{1}(p)$ uniformly for $p \in P$ and $\left|h_{j}(p)-h_{j}(0)\right| \leqq c \delta^{\alpha-1}$ by Lemma 9. Hence the expression above is bounded by a constant independent of $m$ times

$$
d^{n+\alpha} / \delta^{n+\alpha}+d^{n+1} / \delta^{n+1}
$$

Since $|K| \geqq c d^{n}$ and $\delta(z) \geqq d$ for $z \in K$,

$$
\frac{d^{n+\alpha}}{\delta^{n+\alpha}} \leqq c \int_{K} \frac{\delta^{\alpha}(z)}{\delta^{n+\alpha}} d z \leqq c \int_{K} \frac{\delta^{\alpha}(z)}{|z|^{n+\alpha}} d z
$$

Treating $d^{n+1} / \delta^{n+1}$ in the same way and summing over $m$, we see (2.11) is finite.

Turning to (2.12) we have

$$
\begin{aligned}
& \int_{K}|\omega(p)| \frac{d z}{|z|^{n+\alpha}} \\
& \quad \leqq \int_{K} \frac{d z}{|z|^{n+\alpha}} \int|H(y)|\left|\frac{1}{|y-p|^{n+\beta}}-\frac{1}{|y|^{n+\beta}}-\sum p_{j} \frac{\partial}{\partial y}\left(\frac{1}{|y|^{n+\beta}}\right)\right| d y .
\end{aligned}
$$

The part of (2.13) with integration in the inner integral restricted to $|y|>2|p|$ is majorized by a constant times

$$
\int_{K} \frac{d z}{|z|^{n+\alpha}} \int_{|y|>2|p|}|H(y)| \frac{|p|^{2}}{|y|^{n+\beta+2}} d y .
$$

For $z \in K,|z|$ and $|p|$ are comparable since both are comparable to $\delta$. Hence the last integral is less than a constant times

$$
\int_{K} \frac{d z}{|z|^{n+\alpha}} \int_{|y|>c|z|}|H(y)| \frac{|z|^{2}}{|y|^{n+\beta+2}} d y .
$$

Summing over $m$ and changing the order of integration, we obtain

$$
\int \frac{|H(y)|}{|y|^{n+\beta+2}} d y \int_{|z|<|y| / c} \frac{d z}{|z|^{n-\beta}} \leqq c \int \frac{|H(y)|}{|y|^{n+2}} d y .
$$

Consider then the part of (2.13) with integration in the inner integral extended over $|y|<2|p|$. The parts

and

$$
\int_{K} \frac{d z}{|z|^{n+\alpha}} \int_{|y|<2|p|} \frac{|H(y)|}{|y|^{n+\beta}} d y
$$

$$
\int_{K} \frac{d z}{|z|^{n+\alpha}} \int_{|y|<2|p|}|H(y)| \frac{|p|}{|y|^{n+\beta+1}} d y
$$

can be handled as above-that is, by replacing $|p|$ by $|z|$, summing over $m$ and interchanging the order of integration. 
Consider finally the part

$$
\int_{K} \frac{d z}{|z|^{n+\alpha}} \int_{|y|<2|p|}|H(y)| \frac{d y}{|p-y|^{n+\beta}} .
$$

Let $\bar{K}=\bar{K}_{m}$ be $K$ expanded concentrically $k$ times, $k$ taken large and independent of $m$. The part of (2.14) with inner integration over $\bar{K}$ is less than

$$
\int_{K} \frac{d z}{|z|^{n+\alpha}} \int_{|p-y|<c d} \frac{|H(y)|}{|p-y|^{n+\beta}} d y=O\left(\frac{d^{n}}{\delta^{n+\alpha}}\right) \int_{0}^{c d} \frac{d R(t)}{t^{n+\beta}}
$$

where $R(t)=\int_{|z|<t}|H(p+z)| d z \leqq M t^{n+2}$ uniformly in $t$ and $p \in P$. Integrating by parts we obtain the bound $O\left(d^{n+\alpha} / \delta^{n+\alpha}\right)$ considered earlier.

The remaining part of $(2.14)$ is

$$
\int_{K} \frac{d z}{|z|^{n+\alpha}} \int_{|y| \leqq 2|p|, y \notin R \cup P}|H(y)| \frac{d y}{|p-y|^{n+\beta}} .
$$

Since for $z \in K,|z|$ and $|p|$ are comparable and, for $z \in K$ and $y \notin \bar{K},|p-y|$ and $|z-y|$ are comparable, this is less than a constant times

$$
\int_{K} d z \int_{|z-y|>c \delta(y)} \frac{|H(y)|}{|y|^{n+\alpha}} \frac{d y}{|z-y|^{n+\beta}} .
$$

Adding over $m$ and interchanging the order of integration,

$$
\int \frac{H(y)}{|y|^{n+\alpha}} d y \int_{|z-y|>c \delta(y)} \frac{d z}{|z-y|^{n+\beta}} \leqq c \int \frac{|H(y)|}{|y|^{n+\alpha} \delta(y)^{2-\alpha}} d y .
$$

This completes the proof of Theorem 1 for $1<\alpha<2$. The argument for $0<\alpha<1$ is somewhat simpler. The analogues of Lemmas 8 and 9 can be found in [4], and those of Lemmas 3 and 5 are clear. The hypothesis (1.1) is not required in Lemma 1 for $0<\alpha<1$ and is therefore not needed in the argument for $\tilde{g}_{\varepsilon}$. For $\tilde{h}_{\varepsilon}$ one shows that

$$
\int_{E^{n}}|h(x+z)-h(x)| \frac{d z}{|z|^{n+\alpha}}
$$

is finite almost everywhere in $P$ and need not require (1.1).

3. Proof of Theorem 2. We will prove Theorem 2 for $1<\alpha<2$ and begin by recalling several lemmas.

LEMMA 1. Let $u \in L^{p}, 1<p<\infty$, and let $r$ be a nonnegative integer. If $\Omega$ is $a$ spherical harmonic of degree $m \neq 0$, let

$$
v(x)=\text { p.v. } \int u(x-z) \frac{\Omega\left(z^{\prime}\right)}{|z|^{n}} d z,
$$

and let $u(x, \varepsilon)$ and $v(x, \varepsilon), \varepsilon>0$, denote the Poisson integrals of $u$ and $v$. If

$$
\left(\partial^{r} / \partial \varepsilon^{r}\right) u(x, \varepsilon)
$$

has a nontangential limit at every $x \in E \subset E^{n}$ then so has $\left(\partial^{r} / \partial \varepsilon^{r}\right) v(x, \varepsilon)$ almost everywhere in $E$. 
Lemma 3 is a special case of Theorem 7, of [9, p. 173].

Lemma 2. Let $F \in L^{p}, 1 \leqq p<\infty$, and let $F(x, \varepsilon)$ be the Poisson integral of $F$. Suppose $\left(\partial^{r} / \partial \varepsilon^{r}\right) F(x, \varepsilon)$ has a nontangential limit at each $x \in E$. Then given $\varepsilon>0$ there is a closed $P \subset E,|E-P|<\varepsilon$, and a splitting $F=G+H$ such that $G$ has an ordinary rth differential almost everywhere and $H=0$ for $x \in P$.

For a proof, see [10].

LEMMA 3. Let $H \in L^{p}, 1 \leqq p<\infty$. If for each $x$ in a closed set $P, H(x)=0$ and

$$
\left(\varepsilon^{-n} \int_{|z|<\varepsilon}|H(x+z) \pm H(x-z)|^{p} d z\right)^{1 / p}=O\left(\varepsilon^{r}\right),
$$

then for almost all $x \in P$

$$
\left(\varepsilon^{-n} \int_{|z|<\varepsilon}|H(x+z)|^{p} d z\right)^{1 / p}=o\left(\varepsilon^{r}\right)
$$

For a proof see $[12$, p. 91].

LEMMA 4. If $f \in T_{\alpha}^{p}\left(x_{0}\right), 1<\alpha<2,1 \leqq p<\infty$ then $F=J^{\beta} f(\alpha+\beta=2)$ satisfies

$$
\left(\varepsilon^{-n} \int_{|z|<\varepsilon}\left|F\left(x_{0}+z\right)-F\left(x_{0}-z\right)-\sum b_{j} z_{j}\right|^{p} d z\right)^{1 / p}=O\left(\varepsilon^{2}\right)
$$

for some $b_{j}=b_{j}\left(x_{0}\right)$.

Lemma 4 can be proved by the method of [4, pp. 195-197]. Take $x_{0}=0$ and write

$$
F(x)-F(-x)=\int f(z)\left[G_{\beta}(z-x)-G_{\beta}(z+x)\right] d z .
$$

Thus $F(x)-F(-x)$ differs by a linear term in $x$ from

$$
\int\left[f(z)-f(0)-\sum a_{j} z_{j}\right]\left[G_{\beta}(z-x)-G_{\beta}(z+x)\right] d z
$$

We claim that

$$
\int\left[f(z)-f(0)-\sum a_{j} z_{j}\right] G_{\beta}^{(j)}(z) d z
$$

converges absolutely if

$$
\left(\varepsilon^{-n} \int_{|z|<\varepsilon}\left|f(z)-f(0)-\sum a_{j} z_{j}\right|^{p} d z\right)^{1 / p}=O\left(\varepsilon^{n+\alpha}\right) .
$$

It is enough to show the part of the integral over $|z|<1$ converges absolutely. If

$$
\begin{gathered}
R(t)=\int_{|z|<t}\left|f(z)-f(0)-\sum a_{j} z_{j}\right| d z, \\
\int_{|z|<1}\left|f(z)-f(0)-\sum a_{j} z_{j}\right|\left|G_{\beta}^{(j)}(z)\right| d z \leqq c \int_{0}^{1} \frac{d R(t)}{t^{n-\beta+1}} .
\end{gathered}
$$


That this is finite follows by integrating by parts. Hence $F(x)-F(-x)$ differs by a linear term in $x$ from

$$
\begin{aligned}
& \int\left[f(z)-f(0)-\sum a_{j} z_{j}\right]\left[G_{\beta}(z+x)-G_{\beta}(z-x)-2 \sum x_{j} G_{\beta}^{(j)}(z)\right] d z \\
&=\int_{|z|<2|x|}+\int_{|z|>2|x|}=A(x)+B(x) .
\end{aligned}
$$

Here

$$
\begin{aligned}
|B(x)| & \leqq c \int_{|z|>2|x|}\left|f(z)-f(0)-\sum a_{j} z_{j}\right| \frac{|x|^{3}}{|z|^{n-\beta+3}} d z \\
& =c|x|^{3} \int_{2|x|}^{\infty} \frac{d R(t)}{t^{n+\alpha+1}}=O\left(|x|^{2}\right) .
\end{aligned}
$$

The terms of $A(x)$ majorized by

$$
|x| \int_{|z|<2|x|}\left|f(z)-f(0)-\sum a_{j} z_{j}\right|\left|G_{\beta}^{(j)}(z)\right| d z=O(|x|) \int_{0}^{2|x|} \frac{d R(t)}{t^{n-\beta+1}}=O\left(|x|^{2}\right) .
$$

Hence for $|x|<\varepsilon$,

$$
\begin{aligned}
\left|F(x)-F(-x)-\sum b_{j} x_{j}\right| \leqq & c \varepsilon^{2}+\int_{|z|<2 \varepsilon}\left|f(z)-f(0)-\sum a_{j} z_{j}\right| G_{\beta}(z+x) d z \\
& +\int_{|z|<2 \varepsilon}\left|f(z)-f(0)-\sum a_{j} z_{j}\right| G_{\beta}(z-x) d z .
\end{aligned}
$$

For $1 \leqq p<\infty$, Young's theorem implies

$$
\begin{aligned}
& \left(\int_{|x|<\varepsilon}\left|F(x)-F(-x)-\sum b_{j} x_{j}\right|^{p} d x\right)^{1 / p} \\
& \quad \leqq c \varepsilon^{2+n / p}+2\left(\int_{|z|<2 \varepsilon}\left|f(z)-f(0)-\sum a_{j} z_{j}\right|^{p} d z\right)^{1 / p} \int_{|z|<3 \varepsilon} G_{\beta}(z) d z=O\left(\varepsilon^{2+n / p}\right),
\end{aligned}
$$

which proves the lemma.

Let $1 \leqq \alpha<2$ and let $\Omega$ be a spherical harmonic of degree $m \geqq 0, m \neq 1$. In proving Theorems 2 and 3 , it will be convenient to use an approximation $f(x, \varepsilon)$ to

$$
f_{\varepsilon}(x)=\int_{|z|>\varepsilon}[f(x-z)-f(x)] \frac{\Omega\left(z^{\prime}\right)}{|z|^{n+\alpha}} d z
$$

which is harmonic in $(x, \varepsilon)$ for $x \in E^{n}, \varepsilon>0$. For this purpose we define

$$
f(x, \varepsilon)=c_{m}^{(\alpha)} \int_{E^{n}} f(x+z)\left[|z|^{\alpha} \Omega\left(z^{\prime}\right) e^{-\varepsilon|z|}\right]^{\wedge} d z
$$

where $c_{m}^{(\alpha)}$ is an appropriate constant depending only on $\alpha, n$ and $m$. The harmonic function $f(x, \varepsilon)$ is considered in [14] where the following facts are proved. 
LEMMA 5. For $f \in L^{p}, 1 \leqq p<\infty$,

(a) $f(x, \varepsilon)=\int_{E^{n}} f(x+z) K(z, \varepsilon) d z$, where

$$
\begin{aligned}
K(z, \varepsilon) & =\omega_{m}^{(\alpha)} \nu_{m}^{(\alpha)}(\varepsilon /|z|) \Omega\left(-z^{\prime}\right)|z|^{-n-\alpha}, \\
\nu_{m}^{(\alpha)}(r) & =\int_{0}^{\infty} e^{-r s} s^{\gamma+\alpha+1} J_{m+\gamma}(s) d s
\end{aligned}
$$

$J_{v}(s)$ is the Bessel function of order $\nu, \gamma=(n-2) / 2$ and $\omega_{m}^{(\alpha)}$ is a constant depending only on $\alpha, n$ and $m$;

(b) $\left|v_{m}^{(\alpha)}(r)\right| \leqq A r^{-n-\alpha}$;

(c) $\left|\omega_{m}^{(\alpha)} v_{m}^{(\alpha)}(r)-1\right| \leqq A\left[(m r)^{1 / 2}+(m r)^{3 / 2}\right], A=A_{\alpha, n}$;

(d) $\int_{E^{n}} K(z, \varepsilon) d z=0$.

The crucial lemma in proving Theorem 2 is

LEMMA 6. Let $f \in L^{p}, 1 \leqq p<\infty$, and $f \in T_{\alpha}^{p}\left(x_{0}\right), 1<\alpha<2$. If $\lim _{\varepsilon \rightarrow 0} f_{\varepsilon}\left(x_{0}\right)$ exists and is finite then $f(x, \varepsilon)$ is bounded in every cone $\left\{(x, \varepsilon):\left|x-x_{0}\right|<c \varepsilon\right\}$.

Take $x_{0}=0$ and consider

$$
\begin{aligned}
f(0, \varepsilon)-f_{\varepsilon}(0)= & \int_{|z|<\varepsilon}[f(z)-f(0)] K(z, \varepsilon) d z \\
& +\int_{|z|>\varepsilon}[f(z)-f(0)]\left[K(z, \varepsilon)-\frac{\Omega\left(-z^{\prime}\right)}{|z|^{n+\alpha}}\right] d z \\
= & A_{\varepsilon}+B_{\varepsilon} .
\end{aligned}
$$

Here we have used (d) of Lemma 5. Since $f \in T_{\alpha}^{p}(0)$, there are constants $a_{j}$, $j=1, \ldots, n$, such that

$$
R(t)=\int_{|z|<t}\left|f(z)-f(0)-\sum a_{j} z_{j}\right| d z \leqq M t^{n+\alpha} .
$$

Since $\Omega$ is orthogonal to polynomials of degree $1(m \neq 1)$, neither $A_{\varepsilon}$ nor $B_{\varepsilon}$ is changed if we replace $f(z)-f(0)$ in its integrand by $f(z)-f(0)-\sum a_{j} z_{j}$. By (b) of Lemma 5,

$$
\left|A_{\varepsilon}\right| \leqq c \varepsilon^{-n-\alpha} R(\varepsilon)=O(1)
$$

and by (c) of Lemma 5 ,

$$
\left|B_{\varepsilon}\right| \leqq c \int_{\varepsilon}^{\infty}\left(\frac{\varepsilon}{t}\right)^{1 / 2} \frac{d R(t)}{t^{n+\alpha}}
$$

Integrating by parts, $B_{\varepsilon}$ is bounded.

This shows that $f(0, \varepsilon)$ is bounded. To complete the proof, suppose $(x, \varepsilon)$ satisfies $|x|<c \varepsilon$ and consider

$$
f(x, \varepsilon)-f(0, \varepsilon)=\int[f(z)-f(0)][K(z-x, \varepsilon)-K(z, \varepsilon)] d z
$$


Since $\int K(z, \varepsilon) d z=\int z_{j} K(z, \varepsilon) d z=0\left(^{2}\right)$, also $\int z_{j} K(z-x, \varepsilon) d z=0$ and we can majorize the right side above by

$$
\begin{aligned}
\int_{|z|<2 c \varepsilon} \mid f(z)- & f(0) \\
& -\sum a_{j} z_{j} \mid(|K(z-x, \varepsilon)|+|K(z, \varepsilon)|) d z \\
& \quad \int_{|z|>2 c \varepsilon}\left|f(z)-f(0)-\sum a_{j} z_{j}\right||K(z-x, \varepsilon)-K(z, \varepsilon)| d z \\
=A_{\varepsilon}^{\prime} & +B_{\varepsilon}^{\prime} .
\end{aligned}
$$

As before, $A_{\varepsilon}^{\prime}$ is bounded. To show $B_{\varepsilon}^{\prime}$ is bounded, we must estimate the first order derivatives of $K(z, \varepsilon)$ with respect to $z$. However,

$$
\frac{d}{d r} \nu_{m}^{(\alpha)}(r)=-\int_{0}^{\infty} e^{-r s} s^{\gamma+\alpha+2} J_{m+\gamma}(s) d s .
$$

By an argument like that used for Lemma (1.3) of [14],

$$
\frac{d}{d r} \nu_{m}^{(\alpha)}(r)=O(1)+O\left(r^{s}\right), \quad s>0 .
$$

Hence the first order derivatives of $K(z, \varepsilon)$ are bounded by a constant times $\varepsilon^{s}|z|^{-n-\alpha-1-s}$ for $s \geqq 0$.

Since $|x|<c \varepsilon$,

$$
\left|B_{\varepsilon}^{\prime}\right| \leqq c \varepsilon^{s}|x| \int_{2 c \varepsilon}^{\infty} \frac{d R(t)}{t^{n+\alpha+1+s}} \leqq c \varepsilon^{s+1} \int_{2 c \varepsilon}^{\infty} \frac{d R(t)}{t^{n+\alpha+1+s}}=O(1) .
$$

This proves Lemma 6.

In particular, if $f \in L^{p}, 1 \leqq p<\infty$, and $f \in T_{\alpha}^{p}(x)$ and $\lim _{\varepsilon \rightarrow 0} f_{\varepsilon}(x)$ exists and is finite for $x \in E$, then $f(x, \varepsilon)$ is bounded in each nontangential cone with vertex at a point of $E$. By a well-known theorem of Calderón (see [1]), $f(x, \varepsilon)$ has a nontangential limit at almost every point of $E$. If $F=J^{\beta} f$, we claim this implies that

$$
\int_{E^{n}} F(x+z)\left[|z|^{2} \Omega\left(z^{\prime}\right) e^{-\varepsilon|z|}\right]^{\wedge} d z
$$

has a nontangential limit almost everywhere in $E$.

For if $f$ is infinitely differentiable and has compact support, (3.2) is

$$
\int \hat{F}(z) e^{u(x \cdot z)}|z|^{2} \Omega\left(z^{\prime}\right) e^{-\varepsilon|z|} d z=\int \hat{f}(z) d \hat{\mu}(z) e^{(x \cdot z)}|z|^{\alpha} \Omega\left(z^{\prime}\right) e^{-\varepsilon|z|} d z
$$

by Lemma 2(a) of $\S 2$ with $\lambda=\beta$. The last integral is $f_{1}(x, \varepsilon)$ for the function $f_{1}=f * d \mu$, and the same is true for any $f \in L^{p}, 1 \leqq p<\infty$, by approximating. Hence (3.2) has a nontangential limit almost everywhere in $E$ if both

(a) $f_{1}=f * d \mu \in T_{\alpha}^{p}(x)$ and

(b) $\int_{|z|>\delta}\left[f_{1}(x-z)-f_{1}(x)\right]\left(\Omega\left(z^{\prime}\right) /|z|^{n+\alpha}\right) d z$ converges for almost all $x \in E$.

(2) Since $\alpha>1$, (2) and (3) of Lemma 5 imply $z_{j} K(z, \varepsilon)$ is integrable. 
By Lemma 2 of $\$ 2, f_{1}$ differs from $f$ by the sum of a linear combination of terms $J^{2 k} f, k \geqq 1$, and a term $f * R$ where $R$ has derivatives up to a preassigned order in all $L^{p}, 1 \leqq p \leqq \infty$. Clearly $f$ and $f * R$ satisfy (a) and (b) in $E$. Now $J^{2 k} f \in L_{2}^{p} \subset L_{\alpha}^{p}$. Hence (b) is true for each $J^{2 k} f$ by Lemma 1 of $\$ 2$. For (a) we use the proof of Lemma (1.5) of [14] for $p>1$ and Lemma 4 of $\S 2$ for $p=1$.

Now suppose $f \in T_{\alpha}^{p}(x), 1 \leqq p<\infty, 1<\alpha<2$, for $x \in E$ and each

$$
\int_{|z|>\varepsilon}^{\prime}[f(x-z)-f(x)] \frac{\Omega_{j}\left(z^{\prime}\right)}{|z|^{n+\alpha}} d z
$$

converges for $x \in E$ where $\left\{\Omega_{j}\right\}$ is a normalized basis for the spherical harmonics of a fixed degree $m \neq 1, m=0$ if $p=1$. With $F=J^{\beta} f$, each

$$
\int_{E^{n}} F(x+z)\left[|z|^{2} \Omega_{j}(z) e^{-\varepsilon|z|}\right]^{\wedge} d z
$$

has a nontangential limit almost everywhere in $E$. For smooth $F$ and $m \neq 0$, the last integral is a constant times

$$
\frac{\partial^{2}}{\partial \varepsilon^{2}} \int_{E^{n}}\left(T_{j} F\right)(x+z)\left[e^{-\varepsilon|z|}\right]^{\wedge} d z
$$

where $\left(T_{j} F\right)(x)=$ p.v. $F * \Omega_{j}(x) /|x|^{n}$ (see $\left[3\right.$, p. 906]). Since $T_{j}$ is bounded on $L^{p}$ for $p>1$, the same is true for any $f \in L^{p}, p>1$.

Applying Lemma 1, each

$$
\frac{\partial^{2}}{\partial \varepsilon^{2}} \int_{E^{n}}\left(T_{j}^{2} F\right)(x+z)\left[e^{-\varepsilon|z|}\right]^{\wedge} d z
$$

has a nontangential limit almost everywhere in $E$. Since $\sum_{j} \Omega_{j}^{2}$ is constant (see [6, p. 243(2)]), $\sum T_{j}^{2} F=F$. Hence

$$
\frac{\partial^{2}}{\partial \varepsilon^{2}} \int_{E^{n}} F(x+z)\left[e^{-\varepsilon|z|}\right]^{\wedge} d z
$$

has a nontangential limit almost everywhere in $E$. If $m=0(\Omega \equiv 1)$ the same is true for $1 \leqq p<\infty$.

We now decompose $F$ according to Lemma 2. Theorem 2 will follow if $H \in t_{2}^{p}(x)$ for almost all $x \in P$. Since $F=J^{\beta} f$ and $G$ satisfy the conclusion of Lemma 4 in $P$, so does $H$. Since $H=0$ in $P$,

$$
\left(\varepsilon^{-n} \int_{|z|<\varepsilon}|H(x+z)-H(x-z)|^{p} d z\right)^{1 / p}=O\left(\varepsilon^{2}\right)
$$

for $x \in P$, and Theorem 2 follows from Lemma 3 of this section.

4. Proof of Theorem 3. In this section we will prove Theorem 3 and use the proof to obtain an improvement of Theorem 2. We begin with Theorem 3. Its 
proof is similar to that of Theorem 2, but we need a replacement for Lemma 6 of $\S 3$.

Hence let $f \in L^{p}, 1 \leqq p<\infty$, and suppose

$$
f_{\varepsilon}(x)=\int_{|z|>\varepsilon}[f(x-z)-f(x)] \frac{\Omega\left(z^{\prime}\right)}{|z|^{n+1}} d z
$$

converges at $x=x_{0}$, where $\Omega$ is a spherical harmonic of degree $m \neq 1, m=0$ if $p=1$. We claim that

$$
f\left(x_{0}, \varepsilon\right)=c_{m} \int_{E^{n}} f\left(x_{0}+z\right)\left[|z| \Omega\left(z^{\prime}\right) e^{-\varepsilon|z|}\right]^{\wedge} d z
$$

has a limit as $\varepsilon \rightarrow 0$. Here we write $c_{m}=c_{m}^{(1)}, \omega_{m}=\omega_{m}^{(1)}, \nu_{m}=\nu_{m}^{(1)}$.

For taking $x_{0}=0$,

$$
\begin{aligned}
f(0, \varepsilon)-\tilde{f}_{\varepsilon}(0)= & \omega_{m} \int_{|z|<\varepsilon}[f(z)-f(0)] \nu_{m}\left(\frac{\varepsilon}{|z|}\right) \frac{\Omega\left(-z^{\prime}\right)}{|z|^{n+1}} d z \\
& +\int_{|z|>\varepsilon}[f(z)-f(0)]\left[\omega_{m} \nu_{m}\left(\frac{\varepsilon}{|z|}\right)-1\right] \frac{\Omega\left(-z^{\prime}\right)}{|z|^{n+1}} d z \\
= & A_{\varepsilon}+B_{\varepsilon} .
\end{aligned}
$$

We put

$$
S(t)=\int_{|z|<t}[f(z)-f(0)] \Omega\left(-z^{\prime}\right) d z .
$$

Then $S(t)=O\left(t^{n}\right)$ as $t \rightarrow \infty$ and, since $f_{\varepsilon}(0)$ converges, $S(t)=o\left(t^{n+1}\right)$ as $t \rightarrow 0$. However,

$$
A_{\varepsilon}=\int_{0}^{\varepsilon} S^{\prime}(t) G_{\varepsilon}(t) d t
$$

and

$$
B_{\varepsilon}=\int_{\varepsilon}^{\delta} S^{\prime}(t) H_{\varepsilon}(t) d t+o(1)
$$

for fixed $\delta>\varepsilon$ by Lemma $5(\mathrm{c})$ of $\S 3$. Here of course

$$
G_{\varepsilon}(t)=\omega_{m} \nu_{m}(\varepsilon / t) t^{-n-1}, \quad H_{\varepsilon}(t)=\left[\omega_{m} \nu_{m}(\varepsilon / t)-1\right] t^{-n-1} .
$$

Integrating by parts and applying (b) and (c) of Lemma 5 of $\S 3$,

$$
A_{\varepsilon}=-\int_{0}^{\varepsilon} S(t) G_{\varepsilon}^{\prime}(t) d t+o(1), \quad B_{\varepsilon}=-\int_{\varepsilon}^{\delta} S(t) H_{\varepsilon}^{\prime}(t) d t+o(1) .
$$

To show $A_{\varepsilon}$ and $B_{\varepsilon}$ tend to zero, it is therefore enough to show that for $s>0$

$$
G_{\varepsilon}^{\prime}(t)=O\left(\varepsilon^{-n-1} t^{-1}\right), \quad H_{\varepsilon}^{\prime}(t)=O\left(\varepsilon^{s} t^{-n-2-s}\right) .
$$

The estimate for $H_{\varepsilon}^{\prime}$ follows from (3.1) and Lemma $5(\mathrm{c})$ of $\S 3 . G_{\varepsilon}^{\prime}(t)$ is a combination of $\nu_{m}(\varepsilon / t) t^{-n-2}$ and $(d / d t)\left[\nu_{m}(\varepsilon / t)\right] t^{-n-1}$. The first of these is $O\left(\varepsilon^{-n-1} t^{-1}\right)$ and the second is a constant times

$$
\varepsilon t^{-n-3} \int_{0}^{\infty} e^{-(\varepsilon / t) s} s^{\gamma+3} J_{m+\gamma}(s) d s \leqq c \varepsilon t^{-n-3} \int_{0}^{\infty} e^{-(\varepsilon / t) s} s^{2 \gamma+3} d s,
$$


since $\left|J_{m+\gamma}(s)\right| \leqq c s^{\gamma}$ (see [14], Lemma (1.2)). Changing variables our claim follows -i.e., $f(0, \varepsilon)$ has a limit as $\varepsilon \rightarrow 0$.

Suppose in addition that

$$
\left(\varepsilon^{-n} \int_{|z|<\varepsilon}|f(z)+f(-z)-2 f(0)|^{p} d z\right)^{1 / p}=O(\varepsilon) .
$$

Consider

$$
f(x, \varepsilon)=\int f(z) K(z-x, \varepsilon) d z
$$

where $K$ is defined by Lemma 5 of $\S 3$ for $\alpha=1$. If $m$ is odd then $K(z, \varepsilon)$ is odd in $z$ and

$$
f(-x, \varepsilon)-f(x, \varepsilon)=\frac{1}{2} \int_{E^{n}}[f(z)+f(-z)-2 f(0)][K(z+x, \varepsilon)-K(z-x, \varepsilon)] d z .
$$

If $m$ is even then $K(z, \varepsilon)$ is even in $z$ and

$$
\begin{aligned}
f(x, \varepsilon) & +f(-x, \varepsilon)-2 f(0, \varepsilon) \\
= & \frac{1}{2} \int_{E^{n}}[f(z)+f(-z)-2 f(0)][K(z+x, \varepsilon)+K(z-x, \varepsilon)-2 K(z, \varepsilon)] d z .
\end{aligned}
$$

Using (4.1) and arguing as in the last part of the proof of Lemma 6 above, both (4.2) and (4.3) are bounded in any cone $\{(x, \varepsilon):|x|<c \varepsilon\}$. If, in particular, $f$ satisfies (4.1) for $x \in E$ and $f_{\varepsilon}(x)$ converges for $x \in E$ then, taking subsets of $E$, we may assume that

(a) $f(x, \varepsilon)$ is uniformly bounded in $(x, \varepsilon)$ for $x \in E, 0<\varepsilon<\eta$,

(b) either $f(x+z, \varepsilon)+f(x-z, \varepsilon)$ or $f(x+z, \varepsilon)+f(x-z, \varepsilon)-2 f(x, \varepsilon)$ is uniformly bounded for $x \in E$ and $|z|<\varepsilon$.

By a simple argument, it follows $f(x, \varepsilon)$ is bounded in some cone with vertex at each point of density of $E$, and so $f(x, \varepsilon)$ has a nontangential limit almost everywhere in $E$. Under the hypothesis of Theorem 3, therefore, each

$$
\int_{E^{n}} f(x+z)\left[|z| \Omega_{j}\left(z^{\prime}\right) e^{-\varepsilon|z|}\right]^{\wedge} d z
$$

has a nontangential limit almost everywhere in $E$. If $1<p<\infty$ and $m \neq 0$, it follows from Lemma 1 above as before that

$$
\frac{\partial}{\partial \varepsilon} \int_{E^{n}} f(x+z)\left[e^{-\varepsilon|z|}\right]^{\wedge} d z
$$

has a nontangential limit almost everywhere in $E$. If $m=0$, the same is true for $1 \leqq p<\infty$.

By Lemma 2 of $\S 3$, there is for $\varepsilon>0$ a closed $P \subset E,|E-P|<\varepsilon$, and a splitting $f=g+h$ such that $g \in t_{1}^{p}(x)$ for almost all $x$ and $h=0$ in $P$. Since $f$ and $g$ satisfy (4.1) so does $h$ and Theorem 3 follows from Lemma 3 above. 
Finally, we remark that the proof just given can be modified to prove Theorem 2 under an apparently weaker hypothesis on $f$. In fact, the conclusion of Theorem 2 is valid if we replace the hypothesis that $f \in T_{\alpha}^{p}(x), x \in E$ by the condition

$$
\left(\varepsilon^{-n} \int_{|z|<8}|f(x+z)+f(x-z)-2 f(x)|^{p} d z\right)^{1 / p}=O\left(\varepsilon^{\alpha}\right)
$$

if $0<\alpha<1$ or

$$
\left(e^{-n} \int_{|z|<\varepsilon}\left|f(x+z)-f(x-z)-2 \sum a_{j}(x) z_{j}\right|^{p} d z\right)^{1 / p}=O\left(\varepsilon^{\alpha}\right)
$$

if $1<\alpha<2, x \in E$.

We note here that Lemma 4 of $\S 3$ remains true if the hypothesis $f \in T_{\alpha}^{p}\left(x_{0}\right)$ is replaced by (ii) above for $x=x_{0}$. If instead (i) holds for $x=x_{0}$ its analogue is

$$
\left(\varepsilon^{-n} \int_{|z|<\varepsilon}|F(x+z)+F(x-z)-2 F(x)|^{p} d x\right)^{1 / p}=O(\varepsilon), \quad F=J^{1-\alpha} f .
$$

An unpublished result of Stein states that if (ii) holds for $\alpha=1$ and each $x \in E$ then $f \in t_{1}^{p}(x)$ for almost all $x \in E$. Hence assuming (ii) for $\alpha=1$ does not lead to a strengthening of Theorem 2 .

\section{REFERENCES}

1. A. P. Calderón, On the behavior of harmonic functions at the boundary, Trans. Amer. Math. Soc. 68 (1950), 47-54.

2. - "Lebesgue spaces of functions and distributions" in Partial differential equations, Proc. Sympos. Pure Math., Vol. 4, Amer. Math. Soc., Providence, R. I., 1961, pp. 33-49.

3. A. P. Calderón and A. Zygmund, Singular integral operators and differential equations, Amer. J. Math. 79 (1957), 901-921.

4. - Local properties of solutions of elliptic partial differential equations, Studia Math. 20 (1961), 171-225.

5. - Unpublished lecture notes.

6. A. Erdelyi et al., Higher transcendental functions, Vol. 2, (Bateman manuscript project), McGraw-Hill, New York, 1953.

7. Y. Sagher, On hypersingular integrals with complex homogeneity, Thesis, Univ. of Chicago, Chicago, Ill., 1967.

8. E. M. Stein, The characterization of functions arising as potentials, Bull. Amer. Math. Soc. 67 (1961), 102-104.

9. - On the theory of harmonic functions of several variables. II. Behavior near the boundary, Acta Math. 106 (1961), 137-174.

10. - Singular integrals, harmonic functions, and differentiability properties of functions of several variables, Proc. Sympos. Pure Math., Vol. 10, Amer. Math. Soc., Providence, R. I., 1968.

11. E. M. Stein and A. Zygmund, On the fractional derivatives of functions, Proc. London Math. Soc. 14A (1965), 249-264.

12. M. Weiss, On symmetric derivatives in $L^{p}$, Studia Math. 24 (1964), 89-100.

13. M. Weiss and A. Zygmund, On the existence of conjugate functions of higher order, Fund. Math. 48 (1960), 175-187. 
14. R. L. Wheeden, On hypersingular integrals and Lebesgue spaces of differentiable functions. I, Trans. Amer. Math. Soc. 134 (1968), 421-436.

15. - On hypersingular integrals and Lebesgue spaces of differentiable functions. II, Trans. Amer. Math. Soc. 139 (1969), 37-53.

16. A. Zygmund, Trigonometric series, 2nd ed., Vol. 2, Cambridge Univ. Press, Cambridge, 1959.

Rutgers State University,

New Brunswick, New Jersey 